\title{
Erratum to: Combined QCM-D/GE as a tool to characterize stimuli-responsive swelling of and protein adsorption on polymer brushes grafted onto 3D-nanostructures
}

\author{
Meike Koenig • Tadas Kasputis • Daniel Schmidt • \\ Keith B. Rodenhausen • Klaus-Jochen Eichhorn • \\ Angela K. Pannier • Mathias Schubert • \\ Manfred Stamm • Petra Uhlmann
}

Received: 12 November 2014 / Accepted: 12 November 2014 /Published online: 29 November 2014

(C) Springer-Verlag Berlin Heidelberg 2014

\section{Erratum to: Anal Bioanal Chem}

DOI 10.1007/s00216-014-8154-4

Unfortunately, there were some typographical errors in some references of this article. The corrections are given below:

References

4. Chen T, Ferris R, Zhang J, Ducker R, Zauscher S (2010) Prog Polym Sci 35:94

6. Stratakis E, Mateescu A, Barberoglou M, Vamvakaki M, Fotakis C, Anastasiadis SH (2010) Chem Commun 46:4136 15. Koenig M, Rodenhausen KB, Schmidt D, Eichhorn KJ, Schubert M, Stamm M, Uhlmann P (2013) Part Part Syst Charact 30:931

The online version of the original article can be found at http://dx.doi.org/ 10.1007/s00216-014-8154-4.

M. Koenig $\cdot$ K.-J. Eichhorn $\cdot$ M. Stamm $\cdot$ P. Uhlmann $(\bowtie)$

Leibniz-Institut für Polymerforschung Dresden e.V.,

01069 Dresden, Germany

e-mail: uhlmannp@ipfdd.de

M. Koenig $\cdot$ M. Stamm

Department of Physical Chemistry of Polymer Materials,

Technische Universität Dresden, 01062 Dresden, Germany

T. Kasputis $\cdot$ A. K. Pannier

Department of Biological Systems Engineering,

University of Nebraska-Lincoln, Lincoln, NE 68588, USA

D. Schmidt $\cdot$ M. Schubert $\cdot$ P. Uhlmann

Department of Chemistry, University of Nebraska-Lincoln,

Lincoln, NE 68588, USA

K. B. Rodenhausen

Department of Chemical and Biomolecular Engineering,

University of Nebraska-Lincoln, Lincoln, NE 68588, USA
20. Hawkeye MM, Brett MJ (2007) J Vac Sci Technol A $25: 1317$

24. Kasputis T, Koenig M, Schmidt D, Sekora D, Rodenhausen KB, Eichhorn KJ, Uhlmann P, Schubert E, Pannier AK, Schubert M, Stamm M (2013) J Phys Chem C 117:13971

26. de Groot GW, Demarche S, Santonicola MG, Tiefenauer L, Vancso GJ (2014) Nanoscale 6:2228

27. Rodenhausen KB, Schubert M (2011) Thin Solid Films 519:2772

31. Rodenhausen KB, Schmidt D, Kasputis T, Pannier AK, Schubert E, Schubert M (2012) Opt Express 20:5419

T. Kasputis • D. Schmidt $\cdot$ K. B. Rodenhausen • A. K. Pannier •

M. Schubert

Center for Nanohybrid Functional Materials,

University of Nebraska-Lincoln, Lincoln,

NE 68588, USA

M. Schubert

Nebraska Center for Materials and Nanoscience,

University of Nebraska-Lincoln, Lincoln,

NE 68588, USA

Present Address:

M. Koenig

Institute of Functional Interfaces, Karlsruhe Institute of Technology,

76344 Eggenstein-Leopoldshafen, Germany

Present Address:

D. Schmidt

Singapore Synchrotron Light Source, National University of

Singapore, Singapore 117603, Singapore 
32. Schmidt D, Schubert M (2013) J Appl Phys 114:083510

41. Iyer KS, Zdyrko B, Malz H, Pionteck J, Luzinov I (2003) Macromolecules 36:6519
45. Bittrich E, Burkert S, Müller M, Eichhorn KJ, Stamm M, Uhlmann P (2012) Langmuir 28:3439

49. Norde W (2008) Colloids and Surfaces B: Biointerfaces 61:1 